\title{
Primera cita de Acledra nazgul Faúndez, Rider \& Carvajal, 2016 (Heteroptera: Pentatomidae) en La Patagonia
}

\author{
First record of Acledra nazgul Faúndez, Rider \& Carvajal, 2016 \\ (Heteroptera: Pentatomidae) from Patagonia
}

Eduardo I. Faúndez ${ }^{1}$ \& Mariom A. Carvajal ${ }^{1}$

\begin{abstract}
Resumen
Se cita por primera vez al pentatómido Acledra nazgul en la Patagonia, con registros provenientes de la Provincia de Neuquén, Argentina. Se incluye además una nueva cita en la Provincia de La Rioja que llena el vacío distribucional, dado que no existían citas de esta especie en la zona, a pesar de encontrarse en el centro de su distribución. Finalmente, se provee una clave para la identificación de las especies de Acledra en la Patagonia.
\end{abstract}

\section{Palabras clave:}

Pentatominae, Carpocorini, nuevo registro, Argentina, clave.

\begin{abstract}
The stink bug Acledra nazgul is recorded for the first time in Patagonia. The records are coming from the Neuquén Province in Argentina. Additionally, a new record from La Rioja Province is given to fill the distributional gap, as there were no records in the area, which is in between its distribution range. Finally, a key to the Acledra species present in Patagonia is provided.
\end{abstract}

\section{Key words:}

Pentatominae, Carpocorini, new record, Argentina, key.

Acledra Signoret, 1864 es un género sudamericano de pentatómidos fitófagos, que en ocasiones son considerados de importancia económica ya que pueden alimentarse de cultivos, o bien ser interceptados en cargamentos y estaciones de cuarentena (Faúndez \& Verdejo, 2010). Hasta hace poco, este género se encontraba divido en dos subgéneros, Acledra s. str. y Neoacledra Faúndez, 2010 (Faúndez et al. 2014). Sin embargo, Faúndez et al. (2017) tras revisar el subgénero Neoacledra, le asignan rango genérico. Con este cambio, Acledra se compone de 12 especies y tres subespecies; distribuidas en Argentina, Bolivia, Brasil, Chile y Uruguay.

Acledra nazgul Faúndez, Rider \& Carvajal, 2016 (Fig. 1) es una especie recientemente descrita, que se distribuye en las provincias argentinas de Salta, Catamarca, Tucumán, Córdoba y Mendoza. No existe información adicional de esta especie desde su descripción original, y los aspectos de su biología y estados inmaduros son hasta el momento desconocidos. A continuación se listan las primeras citas para esta especie en la Patagonia, además de una nueva cita en La Rioja:

Material examinado: ARGENTINA, Provincia de Neuquén, Chos Malal 37²0'S-70¹6'O, 8-XII1983, L.E. Peña leg., $1{ }^{1} 2$ + 9 ; Provincia de la Rioja, Cuesta Miranda, 1-XII-1983, L.E. Peña leg. 1 q (Todo el material se encuentra depositado en la colección del National Museum of Natural History, Smithsonian Institution, Washington DC, USA USNM).

\footnotetext{
Laboratorio de Entomología, Instituto de la Patagonia, Universidad de Magallanes. Av. Bulnes, 01855,

Punta Arenas, Chile. $\$ ed.faundez@gmail.com.
} 
Esta especie se distribuye hacia la cordillera de los Andes en Argentina (Faúndez et al. 2016), estas nuevas citas extienden su distribución hacia el sur en esta misma área, mientras que el registro de la Rioja llena el vacío distribucional existente para esa provincia que se encuentra en el medio de su rango conocido (i.e., quedando distribuida en las provincias de Salta, Catamarca, Tucumán, Córdoba, La Rioja, Mendoza y Neuquén). Dada la antigüedad de las fechas de captura, estos registros probablemente corresponden a la distribución normal de esta especie y no a introducciones recientes, como se ha experimentado con diversos pentatómidos plaga y/o invasivos en el cono sur de Sudamérica en los últimos años (Faúndez, 2018). En la Patagonia se pueden encontrar otras dos especies pertenecientes al genero Acledra, Acledra modesta (Stål, 1859), ampliamente distribuida en toda la Patagonia Argentina y presente en la Región de Aysén en Chile (Faúndez \& Rider, 2014) y Acledra albocostata lueri Faúndez \& Rider, 2016, presente en las provincias argentinas de Chubut y Río Negro, mientras que en Chile se encuentra en la Región de Aysén (Faúndez \& Rider, 2016). Acledra nazgul puede diferenciarse de ambas especies por los caracteres entregados en la siguiente clave:

Clave para las especies de Acledra Signoret presentes en la Patagonia

1(2) Paraclípeos extendiéndose notoriamente sobre el anteclípeo y contiguos anteriormente (Fig. 2).

A. modesta

2(1)Paraclípeoscasien línea conelanteclípeo, nunca contiguos(Fig. 3). ...3

3(4) Carena interna del corio de color marfil bien definida, línea media de color marfil del escutelo bien desarrollada y engrosada anteriormente y parches laterales escutelares casi imperceptibles (Fig. 4)......... A. albocostata lueri

4(3) Carena interna del corio de color marfil ausente, línea media de color marfil del escutelo vestigial y parches laterales escutelares callosos bien definidos y ovoidales (Fig.5). A. nazgul

\section{AGRADECIMIENTOS}

Agradecemos a Marcos Roca-Cusachs y un revisor anónimo sus sugerencias que ayudaron a mejorar el manuscrito. A Thomas Henry por permitirnos el acceso al material depositado en el USNM.

\section{LITERATURA CITADA}

Faúndez, E.I. \& Rider, D.A. (2014). Nuevos registros de Pentatominae Leach, 1815 (Hemiptera: Heteroptera: Pentatomidae) en Chile. Boletín de la Sociedad Entomológica Aragonesa, 55, 159-162.

Faúndez, E.I. \& Rider, D.A. (2016). Notas sobre el complejo de Acledra (Acledra) albocostata (Spinola, 1852) (Hemiptera: Heteroptera: Pentatomidae: Pentatominae). Arquivos Entomolóxicos, 15, 383-389.

Faúndez, E.I., Rider, D.A. \& Carvajal, M.A. (2014). A new species of Acledra s. str. (Hemiptera: Heteroptera: Pentatomidae) from the highlands of Argentina and Bolivia, with a checklist and key to the species of the nominate subgenus. Zootaxa, 3900(1), 127-134.

Faúndez, E.I., Rider D.A. \& Carvajal, M.A. (2016). Sobre la identidad de Acledra (Acledra) gregalis Berg, 1878, y descripción de una especie nueva de Acledra Signoret, 1864 (Hemiptera: Heteroptera: Pentatomidae: Pentatominae). Arquivos Entomoloxicos, 16, 67-72.

Faúndez, E.I., Rider D.A. \& Carvajal, M.A. (2017). Revisión del género Neoacledra Faúndez, 2010 stat. nov. (Heteroptera: Pentatomidae). Arquivos Entomolóxicos, 18, 101-110.

Faúndez, E.I. \& Verdejo, L.M. (2010). La singular morfología de Acledra haematopa (Spinola, 1852) dentro del género Acledra Signoret, 1864 (Hemiptera: Heteroptera: Pentatomidae), un caso de mimetismo batesiano con descripción de un nuevo 
subgénero. Boletín de la Sociedad Entomológica Aragonesa, 46, 77-82.

Faúndez, E.I. (2018). From agricultural to household pest: The case of the painted bug
Bagrada hilaris (Burmeister) (Heteroptera: Pentatomidae) in Chile. Journal of Medical Entomology, 55, 1-4. https://doi. org/10.1093/jme/tjy091

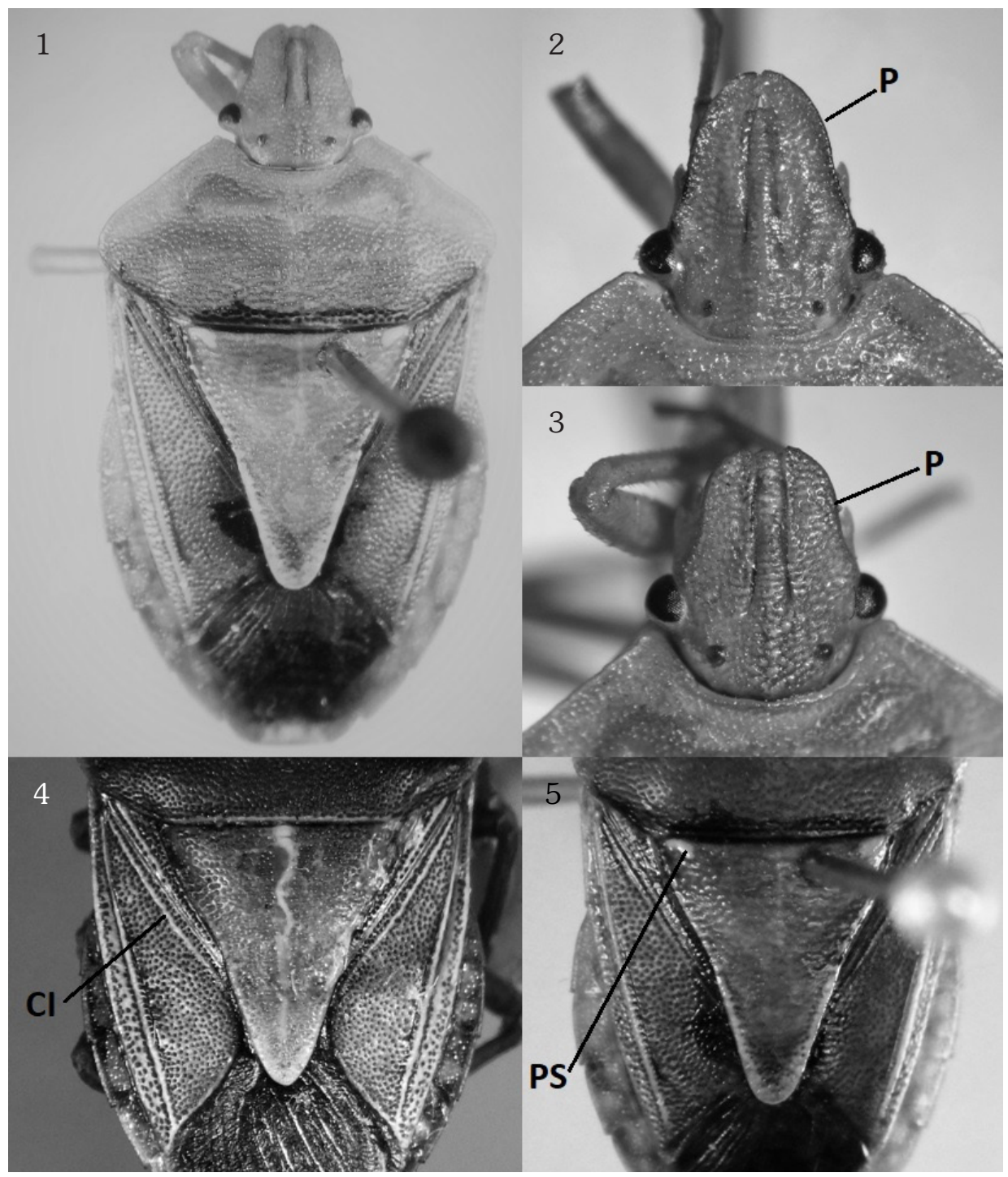

Figs. 1-5. 1. Acledra nazgul, habitus, hembra de Neuquén. 2. Acledra modesta, cabeza. 3. Acledra nazgul, cabeza. 4. Acledra albocostata lueri, detalle de alas y escutelo. 5. Acledra nazgul, detalle de alas y escutelo. $\mathrm{P}$ = paraclípeo; $\mathrm{CI}=$ Carena Interna; $\mathrm{PS}=$ Parche lateral escutelar. 